\title{
Approximate solution of the Fitzhugh-Nagumo equation by the homotopy analysis method
}

\author{
Yinshan \\ College of Sciences of \\ Inner Mongolia University of Technology, \\ Hohhot, 010051, China \\ Email:yinshan@imut.edu.cn
}

\author{
TemuerChaolu \\ College of Sciences and Arts, \\ Shanghai Maritime University, \\ Shanghai, 201306, China \\ Email: tmchaolu@dbc.shmtu.edu.cn
}

\begin{abstract}
In this paper, we used a different initial guess solution then others, to obtain very good approximate solution of the Fitzhugh-Nagumo equation by the homotopy analysis method. Thus, we further illustrate the freedom to select the initial guess solution, i.e. illustrate effectiveness and convenience of homotopy analysis method.
\end{abstract}

Keywords-component: Homotopy analysis method, Fitzhugh-Nagumo equation, Approximate solution

\section{INTRODUCTION}

Nonlinear equations are widely used as models to describe complex physical phenomena in various fields of sciences. The world around us is inherently nonlinear. Nonlinear problems are more difficult to solve than linear ones. Recently, a powerful analytic method for nonlinear problems, the socalled homotopy analysis method (HAM), has been proposed by Liao [1]. Moreover, the homotopy analysis method itself provides us with a convenient way to control the convergence of approximation series and adjust convergence regions when necessary. Briefly speaking, the homotopy analysis method has the following advantages:

1. it is valid even if a given nonlinear problem does not contain any small/large parameters at all;

2. it itself can provide us with a convenient way to control the convergence of approximation series and adjust convergence regions when necessary;

3. it can be employed to efficiently approximate a nonlinear problem by choosing different sets of base functions.

Furthermore, the HAM has been successfully applied to many nonlinear problems such as non-linear eigenvalue problem[2], viscous flows [3], and heat transfer [4], nonlinear oscillations [5], Thomas-Fermi's atom model [6]-[7], the Kawahara equation[8], nano boundary layer flows[9], solitarywave solutions for the fifth-order KdV equation[10], and many other problems(see[11]-[17], for example). And some elegant analytic results are obtained. All of these successful applications of the homotopy analysis method verify its validity for nonlinear problems in science and engineering.

\section{MATHEMATICAL FORMULATION}

The Fitzhugh-Nagumo equation is

$$
u_{t}-u_{x x}=u(u-\alpha)(1-u),
$$

where $\alpha$ is an arbitrary constant. Eq. (1) is an important nonlinear reaction-diffusion equation and applied to model the transmission of nerve impulses[18], [19], also used in biology and the area of population genetics, in circuit theory[20].

Under the transformation $\xi=x-c t$ and $u(x, t)=U(\xi)$, Eq. (1) reads

$$
U^{\prime \prime}+c U^{\prime}+U(U-\alpha)(1-U)=0
$$

where $c$ is the velocity of the travelling wave and the prime denotes the derivative with respect to $\xi$.

We should consider the solitary waves. Obviously, $U(\xi)$ and its derivatives tend to zero as $\xi \rightarrow \infty$. Hence, the boundary conditions of the solitary wave are

$$
U(0)=1, U(+\infty)=0 .
$$

\section{SOlution B Y THE HAM}

S. Abbasbandy[21] solved this problem with same technique, he choose $U_{0}=\exp (-\xi)$ as the initial guess solution. In this paper, we use a different initial guess solution, the concrete procedure is as follows.

According to the Eq. (2) and the boundary conditions (3), the solitary solution can be expressed by

$$
U(\xi)=\sum_{k=1}^{+\infty} b_{k} \exp (-k \xi)
$$

According to the solution expression (4) and the boundary conditions (3), the initial guess solution of $U(\xi)$ is chosen as the following

$$
U_{0}=2 \exp (-\xi)-\exp (-2 \xi) .
$$

Thus, we select the auxiliary linear operator

$$
L[\Phi(\xi, q)]=\frac{\partial^{2} \Phi}{\partial \xi^{2}}+\frac{\partial \Phi}{\partial \xi} .
$$

From (2), we define the nonlinear operator

$$
N[\Phi]=\frac{\partial^{2} \Phi}{\partial \xi^{2}}+C(q) \frac{\partial \Phi}{\partial \xi}-\alpha \Phi+(1+\alpha) \Phi^{2}-\Phi^{3},
$$

Constructing the so-called zeroth-order deformation equation

$$
(1-q) L\left[\Phi(\xi, q)-U_{0}(\xi)\right]=q h H(\xi) N[\Phi(\xi, q)],
$$


subject to the boundary conditions

$$
\Phi(0, q)=1, \Phi(+\infty, q)=0,
$$

where $q \in[0,1]$ is the embedding parameter, $h \neq 0$ is an auxiliary parameter, $H(\xi)$ is an auxiliary function, $\Phi(\xi, q)$ is an unknown function. It is obvious that when the embedding parameter $q=0$ and $q=1$, the equation (7) become

$$
\begin{aligned}
& \Phi(\xi, 0)=U_{0}(\xi), \Phi(\xi, 1)=U(\xi), \\
& C(0)=c_{0}, C(1)=c,
\end{aligned}
$$

respectively. Therefore, when $q$ increases from 0 to 1 , the solution $\Phi(\xi, q)$ varies from the initial guess solution $U_{0}(\xi)$ to the exact solution $U(\xi)$, so does the $C(q)$ from $c_{0}$, the initial guess of the wave speed, to $c$. Expanding $\Phi(\xi, q)$ and $C(q)$ in Taylor series with respect to $q$, we have

$$
\begin{aligned}
& \Phi(\xi, q)=U_{0}(\xi)+\sum_{k=1}^{\infty} U_{k}(\xi) q^{i}, \\
& C(q)=c_{0}+\sum_{k=1}^{\infty} c_{k} q^{k},
\end{aligned}
$$

where

$$
\begin{aligned}
& U_{k}(\xi)=\left.\frac{1}{k !} \frac{\partial^{k} \Phi(x, q)}{\partial q^{k}}\right|_{q=0}, \\
& c_{k}=\left.\frac{1}{k !} \frac{\partial^{k} C(q)}{\partial q^{k}}\right|_{q=0} .
\end{aligned}
$$

The convergence of the series (11)-(12) depends upon the auxiliary parameter $h$. If it is convergent at $\mathrm{q}=1$, we have

$$
\begin{aligned}
& U(\xi)=U_{0}(\xi)+\sum_{k=1}^{\infty} U_{k}(\xi) \\
& c=c_{0}+\sum_{k=1}^{\infty} c_{k}
\end{aligned}
$$

which must be one of the solutions of the original nonlinear equation. Define the vectors

$$
\boldsymbol{U}_{k}=\left\{U_{0}(\xi), U_{1}(\xi), \cdots, U_{k}(\xi)\right\}
$$

Differentiating the zeroth-order deformation Eq.(7) $k$-times with respect to $q$ and then dividing them by $m$ ! and finally setting $q=0$, we obtain the $k$ th-order deformation equation

$$
L\left[U_{k}(\xi)-\chi_{k} U_{k-1}(\xi)\right]=h H(\xi) R_{k}\left(\boldsymbol{U}_{k-1}\right),
$$

subject to

$$
U_{k}(0)=0, U_{k}(+\infty)=0,
$$

where

$$
\begin{aligned}
& R_{k}\left(\boldsymbol{U}_{k-1}\right)=U^{\prime \prime}-\alpha U^{\prime}+\sum_{i=0}^{k-1}\left(c_{k-1-i} U_{i}^{\prime}\right) \\
& +\sum_{i=0}^{k-1}\left((1+\alpha) U_{i} U_{k-1-i}-U_{k-1-n} \sum_{j=0}^{i} U_{j} U_{i-j}\right),
\end{aligned}
$$

and

$$
\chi_{k}= \begin{cases}0, & m \leq 1 \\ 1, & m>1\end{cases}
$$

In $R_{k}\left(\boldsymbol{U}_{k-1}\right)$, the coefficient of $\exp (-\xi)$ is must vanished, otherwise the solution $U_{k}$ of Eq. (17)-(18) should contain the so-called secular term $\xi \exp (-\xi)$. Thus, determining $c_{k-1}$. So, any unknown parameters are not contained in $R_{k}\left(\boldsymbol{U}_{k-1}\right)$.

Let $U_{k}^{*}(\xi)$ denote a special solution of equation

$$
L\left[U_{k}^{*}(\xi)\right]=h H(t) R_{k}\left(\boldsymbol{U}_{k-1}\right) .
$$

Because, the general solution of $L[\phi]=0$ is

$$
\phi=C_{1} \exp (-\xi)+C_{2} .
$$

Thus, the general solution of Eq. (17) can be expressed as

$$
U_{k}(\xi)=\chi_{k} U_{k-1}(\xi)+U_{k}^{*}(\xi)-U_{k}^{*}(0) \exp (-\xi),
$$

In this way, we derive $U_{k}$ for $m=1,2, \cdots$, successively. At the Mth-order approximation, we have the analytic solution of Eq. (2)-(3), namely

$$
U(\xi) \approx W_{M}=\sum_{k=0}^{M} U_{k}(t) .
$$

\section{CONCLusion}

Form (2), we define the error as follow

$$
\begin{array}{cc}
\text { Error } \approx \quad & W_{M}\left(W_{M}-\alpha\right)\left(1-W_{M}\right) \\
& +W_{M}^{\prime \prime}+c W_{M}^{\prime} .
\end{array}
$$

When $\alpha=1.5$, from the $h$-curves, Fig.1., we find that $h \in[-1.4,-0.5]$. If we select $h=-1$, obtain the error of approximate solution as show Fig.2.

When $\alpha=0$, from the $h$-curves, Fig.3., we find that $h \in[-1.1,-0.7]$. If we select $h=-1$, obtain the error of approximate solution as show Fig.4.

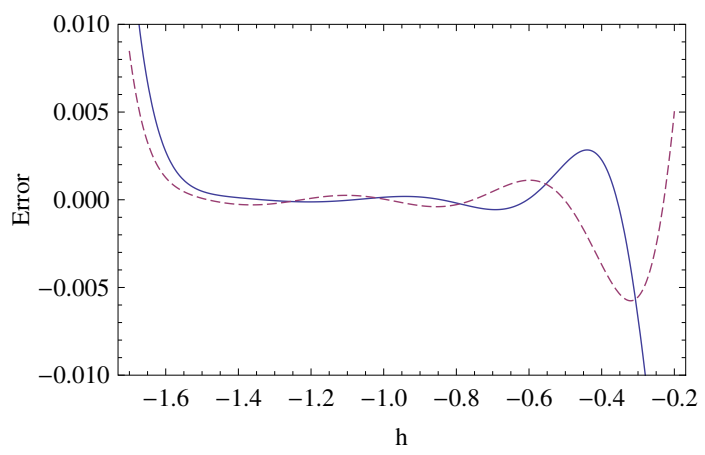

Fig.1. $h$-curves for $a=1.5$, solid line: the error of 15 thorder approximation at $\xi=1$; dashed line: the error of 15 th-order approximation at $\xi=2$. 


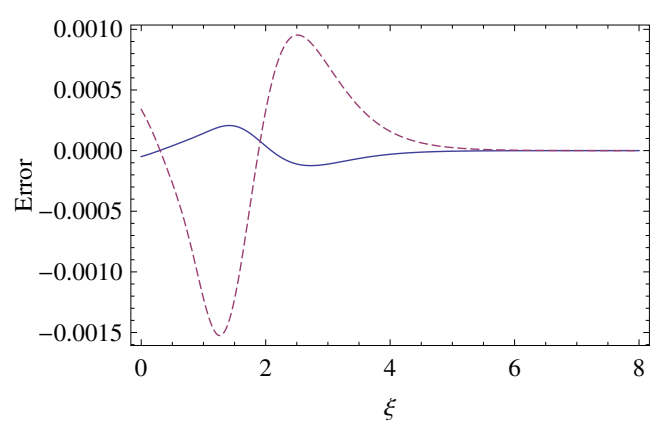

Fig.2. Error for $\alpha=1.5$, solid line: 15th-order analytical approximate solution; dashed line: 10th-order analytical approximate solution.

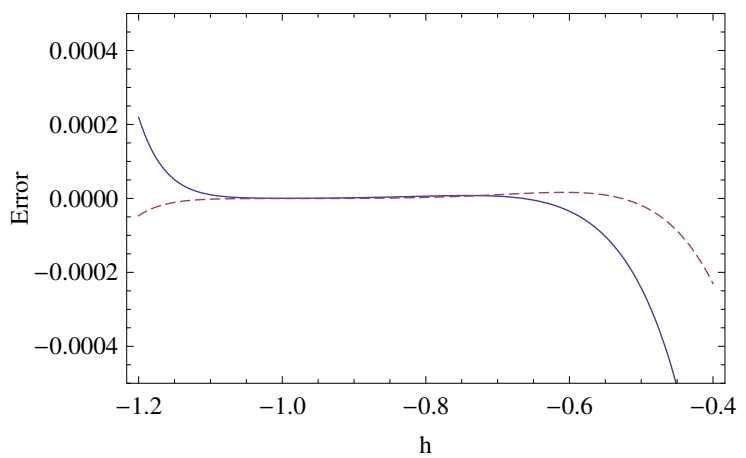

Fig.3. $h$-curves for $a=0$, solid line: the error of 15 th-order approximation at $\xi=1$; dashed line: the error of 15 th-order approximation at $\xi=2$.

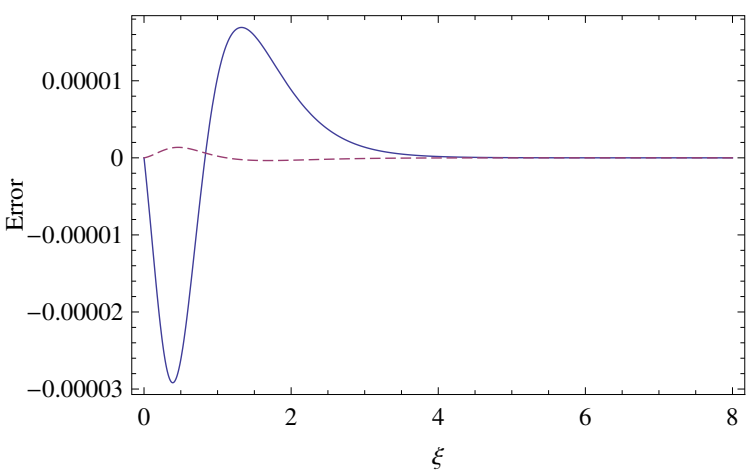

Fig.4. Error for $\alpha=0$, solid line: 10th-order analytical approximate solution; dashed line: 15th-order analytical approximate solution.

\section{ACKNOWLEDGMENT}

This research has been partially Supported by Special Foundation of Doctoral Unit of The Ministry of Education of China(No.20070128001), by Scientific Research innovation Project of Shanghai Education Committee(No. 09YZ239), by Science Project of Inner Mongolia University of Technology (No.X200935).

\section{REFERENCES}

[1] S.J. Liao, The proposed homotopy analysis technique for the solution of nonlinear problems, Ph.D. Thesis, Shanghai Jiao Tong University, 1992.

[2] S.J. Liao, Beyond Perturbation: Introduction to the Homotopy Analysis Method, Science Press in China, 2004.
[3] S.J. Liao, A. Campo, Analytic solutions of the temperature distribution in Blasius viscous flow problems, J. Fluid Mech. vol.453, 2002, pp.411-425.

[4] S. Abbasbandy, The application of homotopy analysis method to nonlinear equations arising in heat transfer, Phys. Lett. A, vol.360, 2006, pp.109-113

[5] S.J. Liao, A.T. Chwang, Application of Homotopy Analysis Method in Nonlinear Oscillations, ASME J. Appl. Mech. vol.65, 1998, pp.914-922.

[6] S.J. Liao, An explicit analytic solution to the Thomas-Fermi equation, Appl. Math. Comput. vol.144, 2003, pp.495-506.

[7] Baoheng Yao, A series solution to the Thomas-Fermi equation, Appl. Math. Comput. vol.203, 2008, pp.396-401.

[8] S. Abbasband, Homotopy analysis method for the Kawahara equation , Nonlinear Anal. RWA. vol.11, 2010, pp.307-312.

[9] Jun Cheng, Shijun Liao, R.N. Mohapatra b, K. Vajravelub, Series solutions of nano boundary layer flows by means of the homotopy analysis method, J. Math. Anal. Appl. vol.343, 2008, pp.233-245.

[10] S. Abbasbandy, F. Samadian Zakaria, Solitary wave solutions to the Kuramoto-Sivashinsky equation by means of the homotopy analysis method, Nonlinear Dynam. vol.51, 2008, pp.83-87.

[11] Cheng-shi Liu, The essence of the homotopy analysis method, Appl.Math.Comput. vol.216, 2010, pp.1299-1303.

[12] S.S. Motsa, P. Sibanda, F.G. Awad, S. Shateyi, A new spectral-homotopy analysis method for the MHD JefferyCHamel problem, Comput. Fluids, vol.39, 2010, pp.1219-1225.

[13] T. Hayat, M. Sajid, On analytic solution for thin film flow of a fourth grade fluid down a vertical cylinder, Phys. Lett. A vol.361, 2007, pp.316322.

[14] Mohammad Mehdi Rashidi, Saeed Dinarvand, Purely analytic approximate solutions for steady three-dimensional problem of condensation film on inclined rotating disk by homotopy analysis method, Nonlinear Anal. RWA, vol.10 , 2009, pp.2346-2356.

[15] Mohammad Zurigat, Shaher Momani, Zaid Odibat, Ahmad Alawneh, The homotopy analysis method for handling systems of fractional differential equations, Appl. Math. Model. vol.34, 2010, pp.24-35.

[16] A.K. Alomari, M.S.M. Noorani, R. Nazar, C.P. Li, Homotopy analysis method for solving fractional Lorenz system, Commun. Nonlinear Sci. Numer. Simul., vol.15, 2010, pp.1864-1872.

[17] Robert A. Van Gorder, K. Vajravelu, On the selection of auxiliary functions, operators, and convergence control parameters in the application of the Homotopy Analysis Method to nonlinear differential equations: A general approach, Commun. Nonlinear Sci. Numer. Simul. vol.14, 2009, pp.4078-4089.

[18] R. Fitzhugh, Impulse and physiological states in models of nerve membrane, Biophys. J. vol.1, 1961, pp.445-466.

[19] J.S. Nagumo, S. Arimoto, S. Yoshizawa, An active pulse transmission line simulating nerve axon, Proc. IRE vol.50, 1962, pp.2061-2070.

[20] M. Shih, E. Momoniat, F.M. Mahomed, Approximate conditional symmetries and approximate solutions of the perturbed Fitzhugh- Nagumo equation, J. Math. Phys. vol.46, 2005, pp.023503.

[21] S. Abbasbandy, Soliton solutions for the Fitzhugh-Nagumo equation with the homotopy analysis method, Appl.Math.Model. vol.32, 2008, pp.2706-2714. 